\title{
Article \\ Nanopore Sequencing and Hi-C Based De Novo Assembly of Trachidermus fasciatus Genome
}

\author{
Gangcai Xie ${ }^{1, *(\mathbb{C}}, \mathrm{Xu}$ Zhang ${ }^{2,3}$, Feng $\mathrm{Lv}^{4}$, Mengmeng Sang ${ }^{1}$, Hairong $\mathrm{Hu}^{5}{ }^{5}$, Jinqiu Wang ${ }^{5, *}$ and Dong $\mathrm{Liu}^{2,3, *(\mathbb{D})}$ \\ 1 Institute of Reproductive Medicine, Medical School, Nantong University, Nantong 226001, China; \\ sangmm12345@163.com \\ 2 Nantong Laboratory of Development and Diseases, School of Life Science, Nantong University, \\ Nantong 226001, China; zhangxu0213@yeah.net \\ 3 Key Laboratory of Neuroregeneration of Jiangsu and Ministry of Education, \\ Co-Innovation Center of Neu-Roregeneration, Nantong University, Nantong 226001, China \\ 4 Nantong College of Science and Technology, Qingnian Middle Road 136, Nantong 226006, China; \\ prlf2019@163.com \\ 5 State Key Laboratory of Genetic Engineering, Institute of Genetics, School of Life Sciences, Fudan University, \\ Shanghai 200438, China; hrhu@fudan.edu.cn \\ * Correspondence: gangcai@ntu.edu.cn (G.X.); jqwang@fudan.edu.cn (J.W.); tom@ntu.edu.cn (D.L.)
}

check for updates

Citation: Xie, G.; Zhang, X.; Lv, F.; Sang, M.; Hu, H.; Wang, J.; Liu, D. Nanopore Sequencing and $\mathrm{Hi}-\mathrm{C}$ Based De Novo Assembly of Trachidermus fasciatus Genome. Genes 2021, 12, 692. https://doi.org/ $10.3390 /$ genes 12050692

Academic Editor: Antonio Figueras

Received: 18 March 2021

Accepted: 27 April 2021

Published: 6 May 2021

Publisher's Note: MDPI stays neutral with regard to jurisdictional claims in published maps and institutional affiliations.

Copyright: (c) 2021 by the authors. Licensee MDPI, Basel, Switzerland. This article is an open access article distributed under the terms and conditions of the Creative Commons Attribution (CC BY) license (https:// creativecommons.org/licenses/by/ $4.0 /)$.
Abstract: Trachidermus fasciatus is a roughskin sculpin fish widespread across the coastal areas of East Asia. Due to environmental destruction and overfishing, the population of this species is under threat. In order to protect this endangered species, it is important to have the genome sequenced. Reference genomes are essential for studying population genetics, domestic farming, and genetic resource protection. However, currently, no reference genome is available for Trachidermus fasciatus, and this has greatly hindered the research on this species. In this study, we integrated nanopore long-read sequencing, Illumina short-read sequencing, and Hi-C methods to thoroughly assemble the Trachidermus fasciatus genome. Our results provided a chromosome-level high-quality genome assembly with a predicted genome size of $542.6 \mathrm{Mbp}(2 n=40)$ and a scaffold N50 of $24.9 \mathrm{Mbp}$. The BUSCO value for genome assembly completeness was higher than $96 \%$, and the single-base accuracy was $99.997 \%$. Based on EVM-StringTie genome annotation, a total of 19,147 protein-coding genes were identified, including 35,093 mRNA transcripts. In addition, a novel gene-finding strategy named RNR was introduced, and in total, 51 (82) novel genes (transcripts) were identified. Lastly, we present here the first reference genome for Trachidermus fasciatus; this sequence is expected to greatly facilitate future research on this species.

Keywords: Trachidermus fasciatus; genome assembly; novel gene; nanopore; Hi-C

\section{Introduction}

The roughskin sculpin (Trachidermus fasciatus) is a small, carnivorous, and catadromous fish that has been found across the coastal areas of China, Korea, and Japan [1,2]. Historically, roughskin sculpin has been named one of the four most famous fishes in China and has been treated as a valuable food source by the Chinese population [3]. However, the population size of this fish species has declined significantly during the past decades due to overfishing and environmental changes in spawning and habitat site [1,4]. Since 1988, roughskin sculpin has been listed as a Class II protected animal by the Chinese government, which encouraged the development of a farming system for its domestication [5]. It had been reported that the karyotype of this species is $2 n=40$ ([6]), and more recently, the genetic diversity [7] and genomic signature [8] of Trachidermus fasciatus had also been studied, which might be important for its conservation management. A reference genome of this species is important for both the development of a farming system and future genetic studies. Although its mitochondrial genome has been previously identified [9], the nuclear genome sequence is still unavailable. 
During the past decades, high-throughput DNA sequencing technologies have advanced significantly, including Illumina short-read sequencing, Pacific biosciences, and Oxford nanopore long-read sequencing $[10,11]$. It has been shown that the nanopore long-read sequencing technology can be used for genome assembly in different species, including bacterial [12], human [13,14], and rice [15]. Furthermore, chromosome-scale assemblies of human and mouse genomes can be generated by integrating short-read DNA sequencing and $\mathrm{Hi}-\mathrm{C}$ chromatin interaction mate-pair sequencing [16]. By combining Hi-C and short-read data, a scaffold spatial orienting accuracy of $99 \%$ was achieved [16].

In this study, Oxford nanopore sequencing, Illumina short-reads sequencing technologies, and the Hi-C method were integrated for de novo genome assembly of Trachidermus fasciatus (Figure 1). This study not only provides the first high-quality reference genome for the communities studying roughskin sculpin but also identified 51 novel genes and 82 novel transcripts.

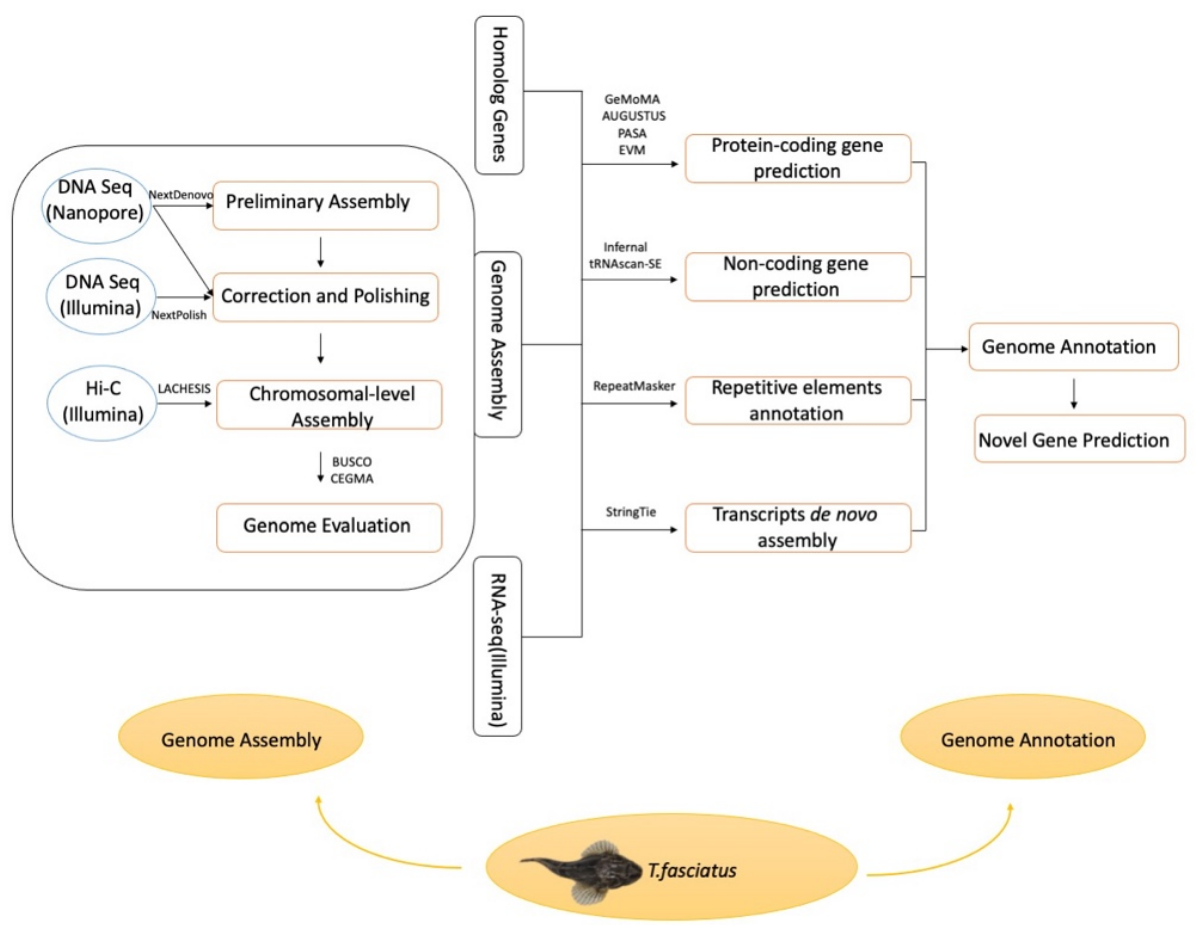

Figure 1. Pipeline of Trachidermus fasciatus genome assembly and annotation.

\section{Materials and Methods}

\subsection{Tissue Extraction and Sequencing}

A single 1 year old live Trachidermus fasciatus (NCBI:txid290630) fish was collected for tissue extraction. The study was conducted following Chinese law for the Protection of Animals, and the animal was treated properly and in line with the ARRIVE guidelines. Tissues from seven different areas were collected for RNA sequencing, including liver, gall bladder, stomach, heart, kidney, gill, and skin. The muscle samples were collected for both of DNA sequencing (nanopore and Illumina sequencing) and Hi-C sequencing (Illumina sequencing). The tissue samples were stored in liquid nitrogen before sequencing. Trachidermus fasciatus genomic DNA was extracted using the QIAGEN@Genomic DNA extraction kit (Cat\#13323, Qiagen, Valencia, CA, USA) following manufacturer's instructions. An RNA sequencing library was constructed using the Illumina TruSeq RNA library preparation kit. Nanopore sequencing was carried on a Nanopore GridION X5/PromethION sequencer (ONT, Oxford, UK), while Illumina sequencing (DNA, RNA, Hi-C) was performed using the Illumina HiSeq platform (Illumina, San Diego, CA, USA). Finally, the Hi-C library preparation was performed according to a previously reported protocol [17]. 


\subsection{De Novo Genome Assembly}

NextDenovo was used for genome assembly, including sequencing error correction, preliminary assembly, and genome polishing. The NextCorrect module was used for raw read correction and consensus sequence extraction. The NextGraph module was used for preliminary assembly, and the NextPolish module was used for genome polishing [18]. At the genome polishing stage, nanopore reads were used repetitively three times, and Illumina sequencing reads were used four times for genome correction. The seed cutoff was set at $38 \mathrm{Kbp}$, and the read cutoff was set at $1 \mathrm{Kbp}$ for the NextDenovo genome assembly, while default parameters were used for other settings.

\subsection{Quality Evaluation of Genome Assembly}

To evaluate the quality of the genome assembly, four evaluation metrics were applied, including genome completeness, genome accuracy and consensus, GC proportion and sequencing-depth distribution (GC-depth analysis), and genome contamination. Both BUSCO [19] and CEGMA [20] were used for genome completeness evaluation. BUSCO evaluated the completeness of the assembly by matching it with the ortholog genes from the OrthoDB [21] database (actinopterygii_odb9), and the evaluation of CEGMA was performed by comparing the evolutionarily conserved core protein-coding genes in eukaryotes ( 248 core genes). To assess the assembled genome sequence accuracy and consensus, Illumina sequencing reads were mapped onto the genome by Burrows-Wheeler aligner (BWA) [22]. Samtools and bcftools [23] were used for SNP and Indel calculations. The percentage of homogeneous SNPs was considered as the single nucleotide error rate of the assembled genome. For the GC-depth analysis, nanopore sequencing reads were mapped onto the genome assembly using minimap2 [24], and the GC content proportion and long read coverage were calculated for each sliding window (size of $10 \mathrm{kbp}$ ) of the assembled genome. Finally, the assembled genome was compared to the sequences from nucleotide sequence database (NT, ftp.ncbi.nih.gov/blast/db) to examine the interspecies contamination. The genome was divided into $1 \mathrm{Mbp}$ bins and then aligned with the NT sequences using the blastn [25] software. The mapping statistics were summarized based on the results from each bin.

\subsection{Hi-C Guided Genome Assembly}

The raw paired-end Hi-C reads were preprocessed by fastp [26] for adapter trimming and low-quality read filtering (only keeping reads with Phred Score $>15$, and $5>$ number of Ns in the reads). Each pair of clean reads was mapped onto the assembled genome using bowtie2 [22] (version: 2.3.2, parameters: -end-to-end,-version-sensitive -L 30). For the reads that could be mapped onto the genome, the DpnII restriction endonuclease recognition sequence pattern GATC was searched, and the reads were cut at their restriction sites and used for further mapping. Each pair of uniquely mapped reads was merged for further analysis. LACHESIS [16] (parameters: CLUSTER MIN RE SITES = 100; CLUSTER MAX LINK DENSITY $=2.5$; CLUSTER NONINFORMATIVE RATIO $=1.4 ;$ ORDER MIN N RES IN TRUNK = 60; ORDER MIN N RES IN SHREDS $=60$ ) was used to obtain chromosomelevel scaffolds based on the primary assembly and the Hi-C read mapping information. To draw inter- and intra-chromosomal Hi-C interaction heatmap, each chromosome was divided into bins with length of $100 \mathrm{Kbp}$, and coverage of Hi-C reads pairs for each pair of bins was calculated and treated as the linkage intensity. The linkage intensity for each bin pair was further natural log transformed for heatmap representation.

\subsection{Gene and Repetitive Element Annotation}

Based on the RNA sequencing data from eight tissues, the assembled genome, and public homolog protein sequences, Trachidermus fasciatus genome was annotated at different levels, including its repetitive elements, non-protein-coding RNAs (ncRNA), and proteincoding genes. First, RepeatMasker [27] was applied to annotate the repetitive elements (RE), and the repeats masked genome was further used for gene annotation. To construct 
RE database for RepeatMasker, MITE-hunter [28] was used to search miniature invertedrepeat transposable elements (MITE DB) in reference assembly. The reference assembly was hard masked by MITE DB through RepeatMasker, and then RepeatModeler was used to search RE sequences in de novo (RM DB) based on MITE hard masked reference assembly. In addition, the MITE DB, RM DB, and Repbase [29] (Repbase-derived RepeatMasker libraries RELEASE 20181026) were combined into one repetitive elements database (RE $\mathrm{DB})$, which was further used for RepeatMasker prediction of REs. For the protein-coding gene annotation, at the first stage, EVM [30] was used to integrate the annotation results from three methods, including transcriptome prediction by PASA [31], homolog protein predicted by GeMoMa [32], and de novo gene predicted by AUGUSTUS [33]. At the second stage, transcripts were assembled by StringTie by sequentially five procedures. Firstly, RNA-seq reads were mapped onto Trachidermus fasciatus genome by hisat2 [34] (Table 1 for mapping statistics), and then the primary transcripts were assembled by StringTie [35] without guide reference annotation for each tissue individually. Thirdly, the GTF files from the transcripts assembly of each tissue were merged by StringTie. Fourthly, the transcripts were assembled by StringTie with merged GTF file as guide reference annotation for each tissue individually. Finally, the GTFs from the second round of transcripts assembly were merged as StringTie transcripts. At the third stage, the transcripts from EVM integration and StringTie assembly were further merged by StringTie, and the coding regions of EVM-StringTie merged transcripts were predicted by TransDecoder, where the length of protein amino acids was required to be longer than 100. The coding sequences were further filtered by Gffread [36], where the transcripts lacking initial start codon, the terminal stop codon, or having an in-frame stop codon were discarded. Furthermore, Infernal [37] and tRNAscan-SE [38] were used to predict ncRNAs and tRNAs, respectively.

Table 1. Hisat2 mapping statistics for the RNA-seq datasets of seven tissues.

\begin{tabular}{ccccccc}
\hline Sample & Number of Bases & $\begin{array}{c}\text { Total (Pairs } \\
\text { of Reads) }\end{array}$ & $\begin{array}{c}\text { Mapped } \\
\text { (Unique) }\end{array}$ & $\begin{array}{c}\text { Mapped } \\
\text { (Multiple) }\end{array}$ & Mapped (All) & $\begin{array}{c}\text { Mapped } \\
\text { (Percentage) }\end{array}$ \\
\hline skin & $8,250,236,700$ & $27,500,789$ & $22,963,898$ & $2,672,115$ & $25,636,013$ & $93.22 \%$ \\
stomach & $10,785,480,600$ & $35,951,602$ & $26,050,112$ & $7,422,957$ & $33,473,069$ & $93.11 \%$ \\
gill & $8,196,614,400$ & $27,322,048$ & $23,854,282$ & $1,804,852$ & $25,659,134$ & $93.91 \%$ \\
gallbladder & $10,626,212,100$ & $35,420,707$ & $30,789,079$ & $1,382,971$ & $32,172,050$ & $90.83 \%$ \\
kidney & $9,276,090,300$ & $30,920,301$ & $27,633,067$ & $1,376,568$ & $29,009,635$ \\
heart & $8,720,360,400$ & $29,067,868$ & $24,859,462$ & $1,093,286$ & $25,952,748$ & $83.82 \%$ \\
liver & $9,334,292,100$ & $31,114,307$ & $27,014,139$ & $2,174,980$ & $29,189,119$ & $93.28 \%$ \\
Average & $9,312,755,229$ & $31,042,517$ & $26,166,291$ & $2,561,104$ & $28,727,395$ & $92.57 \%$ \\
Total & $65,189,286,600$ & $217,297,622$ & $183,164,039$ & $17,927,729$ & $201,091,768$ \\
\hline
\end{tabular}

\subsection{Novel Transcripts/Gene Discovery}

RNR method (RefSeq-NT-RNASeq integrated novel gene selection) was created to identify novel genes and transcripts in Trachidermus fasciatus, which contains following three steps: model organism RefSeq sequence alignment, NCBI nucleotide database (NT) alignment, and RNA-Seq expression filtering. At model organism sequence alignment step, the RefSeq RNA sequences of four model organisms (Homo sapiens, Mus musculus, Danio rerio, and Caenorhabditis elegans) were downloaded from UCSC Genome Browser (hg38, mm10, danRer11, and ce11). The EVM-StringTie mRNA sequences were aligned to RefSeq RNA sequences by pblat [39] (same as blat but with multithreads support) with the setting of "-threads $=40$-minScore $=30$-minIdentity $=60 "$. At the second step, the nucleotide database (NT) was downloaded from NCBI (ftp.ncbi.nlm.nih.gov:/blast/db/FASTA/nt. $\mathrm{gz}$ ), and then the index of NT was built by "makeblastdb" (from NCBI-BLAST [40]). The mRNA sequences failed to be mapped at the first step were re-aligned onto NT database by blastn (NCBI-BLAST, value was set to be 0.05). At the last step, transcript-level expression values were calculated by salmon [41] software, and then only the unmapped transcripts from the first two steps with at least 50 reads detected in at least one tissue were defined as 
the final novel transcripts. Novel genes were defined as the genes that are associated with the novel transcripts at the final step and also with all of their transcripts unmapped at the first two steps. To study the protein sequence novelty, the predicted protein sequences of novel transcripts were aligned to NCBI NR database by blastp [42] (expected threshold 0.05). For the visualization of the novel genes, ggsashimi [43] was used to generate sashimi plot to show both of RNA-seq reads density and exon-exon junctional linkages on the candidate novel genes.

\subsection{Data Availability and Software Details}

The genome sequence and genome annotation of Trachidermus fasciatus has been stored in the Genome Warehouse of National Genomics Data Center [44], Beijing Institute of Genomics (BIG), Chinese Academy of Sciences, under accession number GWHACFF00000000.2, which is publicly accessible at https:/ /bigd.big.ac.cn/gwh. R was used for graphical plotting, and the source and version of the software used are listed in Table 2 below.

Table 2. The sources and versions of the software used.

\begin{tabular}{|c|c|c|}
\hline Software & Version & Source Link \\
\hline Augustus & v3.3.1 & $\begin{array}{c}\text { https:/ / github.com/Gaius-Augustus / Augustus } \\
\text { accessed on } 3 \text { October } 2018\end{array}$ \\
\hline Bcftools & v1.8.0 & $\begin{array}{c}\text { http:/ / samtools.github.io/bcftools / } \\
\text { accessed on } 28 \text { April } 2018\end{array}$ \\
\hline blast & v2.9 & $\begin{array}{l}\text { ftp: / /ftp.ncbi.nlm.nih.gov/blast/executables/blast+/LATEST/ } \\
\text { accessed on } 10 \text { April } 2019\end{array}$ \\
\hline BUSCO & 3.1 .0 & $\begin{array}{c}\text { https://busco.ezlab.org/ } \\
\text { accessed on } 3 \text { October } 2018\end{array}$ \\
\hline BWA & 0.7.12-r1039 & $\begin{array}{l}\text { https://github.com/lh3/bwa } \\
\text { accessed on } 15 \text { January } 2016\end{array}$ \\
\hline CEGMA & v2 & $\begin{array}{l}\text { https:/ / github.com/KorfLab/CEGMA_v2/ } \\
\text { accessed on } 3 \text { October } 2018\end{array}$ \\
\hline EVidenceModeler & v1.1.1 & $\begin{array}{l}\text { http: / / evidencemodeler.github.io/ } \\
\text { accessed on } 4 \text { October } 2018\end{array}$ \\
\hline fastp & 0.19 .4 & $\begin{array}{l}\text { https: / / github.com/OpenGene/ fastp } \\
\text { accessed on } 3 \text { October } 2018\end{array}$ \\
\hline GeMoMa & v1.6.1 & $\begin{array}{l}\text { http:/ / www.jstacs.de/index.php/GeMoMa } \\
\text { accessed on } 28 \text { October } 2019\end{array}$ \\
\hline GMATA & v2.2 & $\begin{array}{c}\text { https:/ / sourceforge.net/projects / gmata / } \text { source=navbar } \\
\text { accessed on } 3 \text { October } 2018\end{array}$ \\
\hline Infernal & v1.1.2 & $\begin{array}{l}\text { http:/ / eddylab.org/infernal/ } \\
\text { accessed on } 2 \text { October } 2018\end{array}$ \\
\hline Minimap2 & 2.17(r941) & $\begin{array}{l}\text { https: / / github.com/lh3/minimap2 } \\
\text { accessed on } 3 \text { June } 2019\end{array}$ \\
\hline NextDenovo & v2.0-beta.1 & $\begin{array}{l}\text { https://github.com/Nextomics/NextDenovo.git } \\
\text { accessed on } 20 \text { October } 2018\end{array}$ \\
\hline NextPolish & v1.0.5 & $\begin{array}{l}\text { https:/ / github.com/Nextomics/NextPolish.git } \\
\text { accessed on } 20 \text { October } 2018\end{array}$ \\
\hline PASA & v2.3.3 & $\begin{array}{c}\text { https: / / github.com/PASApipeline/PASApipeline } \\
\text { accessed on } 3 \text { October } 2018\end{array}$ \\
\hline $\mathbf{R}$ & V3.5.2 & $\begin{array}{c}\text { https: / / www.r-project.org/ } \\
\text { accessed on } 2 \text { January } 2019\end{array}$ \\
\hline RepeatMasker & Revision 1.331 & $\begin{array}{c}\text { https:/ / github.com/rmhubley/RepeatMasker } \\
\text { accessed on } 1 \text { October } 2018\end{array}$ \\
\hline Samtools & v1.4 & $\begin{array}{l}\text { https: / / github.com/samtools / samtools } \\
\text { accessed on } 1 \text { May } 2017\end{array}$ \\
\hline tRNAscan-SE & $\mathrm{v} 2.0$ & $\begin{array}{l}\text { http:/ / lowelab.ucsc.edu/tRNAscan-SE/ } \\
\text { accessed on } 3 \text { October } 2019\end{array}$ \\
\hline Hisat2 & $\mathrm{v} 2.2 .1$ & $\begin{array}{c}\text { https:/ / github.com/DaehwanKimLab / hisat2 } \\
\text { accessed on } 21 \text { September } 2020\end{array}$ \\
\hline StringTie & $\mathrm{v} 2.1 .4$ & $\begin{array}{l}\text { https: / / github.com/gpertea/stringtie } \\
\text { accessed on } 20 \text { July } 2020\end{array}$ \\
\hline
\end{tabular}


Table 2. Cont.

\begin{tabular}{|c|c|c|}
\hline Software & Version & Source Link \\
\hline TransDecoder & v5.5.0 & $\begin{array}{l}\text { https://github.com/TransDecoder } \\
\text { accessed on } 21 \text { September } 2020\end{array}$ \\
\hline gffread & v0.12.3 & $\begin{array}{l}\text { https://github.com/gpertea/gffread } \\
\text { accessed on } 21 \text { September } 2020\end{array}$ \\
\hline NCBI-blast & v2.11.0 & $\begin{array}{l}\text { https:/ / ftp.ncbi.nlm.nih.gov/blast } \\
\text { accessed on } 28 \text { November } 2020\end{array}$ \\
\hline salmon & v1.4.0 & $\begin{array}{l}\text { https:/ / github.com/COMBINE-lab/salmon } \\
\text { accessed on } 28 \text { November } 2020\end{array}$ \\
\hline MITE-Hunter & - & $\begin{array}{c}\text { https:/ / github.com/jburnette/MITE-Hunter } \\
\text { accessed on } 3 \text { October } 2018\end{array}$ \\
\hline RepeatModeler & version open-1.0.11 & $\begin{array}{c}\text { https: / / github.com/Dfam-consortium/RepeatModeler } \\
\text { accessed on } 1 \text { October } 2018\end{array}$ \\
\hline
\end{tabular}

\section{Results}

\subsection{Sequencing Data Sets}

Following nanopore sequencing (Table 3), we obtained approximately 4 million reads that passed quality control, containing more than 87 billion nucleotide bases. The longest read was over $240 \mathrm{kbp}$, and the N50 was approximately $30 \mathrm{kbp}$. More than $70 \%$ of the reads had a length exceeding $10 \mathrm{kbp}$, while roughly $12 \%$ of the reads were exceeding $40 \mathrm{kbp}$. For Illumina sequencing, we obtained more than 350 million reads for both genome and Hi-C sequencing (Table 3), which contains more than 50 billion nucleotide bases. For RNA-seq of seven tissues, 434 million paired-end reads were sequenced, containing more than 65 billion bases. Based on the estimated assembly size, nanopore sequencing and Illumina sequencing (genomic DNA) had average genomic coverage of 161X and 100X, respectively (Table 3 ).

Table 3. Summary of sequencing datasets.

\begin{tabular}{|c|c|c|c|c|}
\hline Library & Number of Bases & Number of Reads & Reads Length (Mean, bp) & Reads Length (Max, bp) \\
\hline nanopore-seq-lib1 & $45,424,703,117$ & $2,049,727$ & 22,161 & 240,976 \\
\hline nanopore-seq-lib2 & $41,854,167,498$ & $2,031,772$ & 20,599 & 243,222 \\
\hline Total & $87,278,870,615$ & $4,081,499$ & 21,384 & 243,222 \\
\hline Library & N50 & $>10 \mathrm{~kb}$ Percentage & $>20$ kb Percentage & $>40 \mathrm{~kb}$ Percentage \\
\hline nanopore-seq-lib1 & 31,576 & 75.85 & 48.11 & 13.48 \\
\hline nanopore-seq-lib2 & 30,199 & 70.82 & 42.38 & 11.99 \\
\hline Total & 30,943 & 73.35 & 45.26 & 12.74 \\
\hline Library & Number of Bases & Number of Reads & Reads Length (Mean, bp) & Reads Length (Max, bp) \\
\hline NGS-Genome & $54,380,742,900$ & $362,538,286$ & 150 & 150 \\
\hline NGS-HiC & $60,291,587,700$ & $401,943,918$ & 150 & 150 \\
\hline \multirow[t]{2}{*}{ NGS-RNASeq } & $65,189,286,600$ & $434,595,244$ & 150 & 150 \\
\hline & \multicolumn{2}{|c|}{ Coverage $(X)$ of Predicted Assembly } & \multicolumn{2}{|c|}{ Predicted Genome Size $(b p, 2 n=40)$} \\
\hline Nanopore-seq & \multicolumn{2}{|c|}{160.836} & \multicolumn{2}{|c|}{$542,656,829$} \\
\hline NGS-Genome & \multicolumn{2}{|c|}{100.212} & & \\
\hline
\end{tabular}

\subsection{De Novo Assembly and Genome Polishing}

Trachidermus fasciatus genome was preliminarily assembled based on nanopore sequencing data and then polished based on both of nanopore and Illumina sequencing data (Figure 1). In the preliminary stage, 62 contigs were assembled, with an N50 exceeding $23 \mathrm{Mbp}$. The longest contig was longer than $35 \mathrm{Mbp}$, while the total length of the prelimi- 
nary genome was approximately 539.1 Mbp (Table 4). After polishing with the Illumina short-read sequencing data, the N50 increased from 23.4 to $23.55 \mathrm{Mbp}$, and the full-length assembly reached roughly 542.6 Mbp. Based on N90 information (Table 4), 23 contigs have a combined assembly coverage of $90 \%$, which indicates that the number of N90 contigs (23) is close to the haploid chromosome number of Trachidermus fasciatus based on karyotype $(2 n=40)$ reported previously $[3,6]$.

Table 4. Summary of genome assembly.

\begin{tabular}{ccccc}
\hline & \multicolumn{2}{c}{ Assembly (Preliminary) } & \multicolumn{2}{c}{ Assembly (Polished) } \\
\cline { 2 - 5 } & Contig Length (bp) & Contig Number & Contig Length (bp) & Contig Number \\
\hline N50 & $23,408,022$ & 10 & $23,556,738$ & 10 \\
N60 & $20,522,422$ & 13 & $20,673,841$ & 13 \\
N70 & $18,786,353$ & 16 & $18,887,485$ & 16 \\
N80 & $17,637,255$ & 18 & $17,756,054$ & 18 \\
N90 & $7,606,484$ & 23 & $7,646,988$ & 23 \\
Longest & $35,774,240$ & 1 & $36,041,605$ & 1 \\
Total & $539,115,043$ & 62 & $542,654,729$ & 62 \\
Length $\geq 5 \mathrm{~kb}$ & $539,115,043$ & 62 & $542,654,729$ & 62 \\
\hline
\end{tabular}

\subsection{Genome Quality Evaluation}

The quality of the assembled genome was further evaluated using different methods. First, the GC content and nanopore sequencing depth distribution were examined based on $10 \mathrm{kbp}$ sliding windows. As shown in Figure 2A, only one peak was observed for the GC content and sequencing depth distribution, which indicates no interspecies contamination. Then, the completeness of the assembled genome was evaluated by both CEGMA [20] and BUSCO [19] (Figure 2B,C), and the results indicated a high percentage of completeness (98.39\% and $96.95 \%$, respectively). Next, high-quality Illumina sequencing reads were mapped onto the assembled genome to evaluate its quality, and the results revealed $99.48 \%$ reads mappability with $99.35 \%$ of the assembled genome being covered at least once (Figure 2D). Finally, genome contamination was examined by matching the assembled contigs with known metazoa genome sequences (based on $50 \mathrm{kbp}$ bins). As shown in Figure $2 \mathrm{E}, 98.79 \%$ of the genome length can be matched with known metazoan genome sequences, which indicates no significant contamination from bacteria.

The single-base-level accuracy was examined by mapping the Illumina short reads onto the assembly. At a depth of no less than $5 \times$ genome-level coverage, 3444 homogeneous single nucleotide polymorphisms (SNPs) and 14,558 insertions or deletions (Indels) were found, occupying $0.000635 \%$ and $0.002683 \%$ of the genome, respectively. This gives the assembled genome with an estimated single-base accuracy of $99.9967 \%$.

\subsection{Hi-C Proximity-Guided Assembly of Chromosome-Level Scaffolds}

Compared to the primary assembly comprised of 62 contigs, the Hi-C-enhanced assembly showed longer cumulative scaffolds (Figure 3A). Using Hi-C chromatin interaction data, the contigs were rearranged based on the interaction information, and a total of 44 scaffolds were assembled (Figure 3B, Table S1). Based on the top 20 longest scaffolds, which represent the main chromosomal scaffolds of Trachidermus fasciatus, Hi-C chromatin interaction events were significantly enriched in intrachromosome but not interchromosome regions (Figure 3C). The number of N90 contigs/scaffolds decreased from 23 to 18 (Table S1), and the top 20 scaffolds reached a cumulative length of approximately $533.7 \mathrm{Mbp}$, occupying $98.35 \%$ of the assembled genome (Figure 3D). This indicates that a chromosome-scale genome assembly was achieved after $\mathrm{Hi}-\mathrm{C}$ proximity-guided enhancement. The length in base pairs of each chromosome was illustrated by the barplot in Figure 3E, which shows that the longest chromosome (chr1) of Trachidermus fasciatus has about 48 million base pairs, and the length of shortest chromosome (chr20) is about 15.6 Mbp. 
A

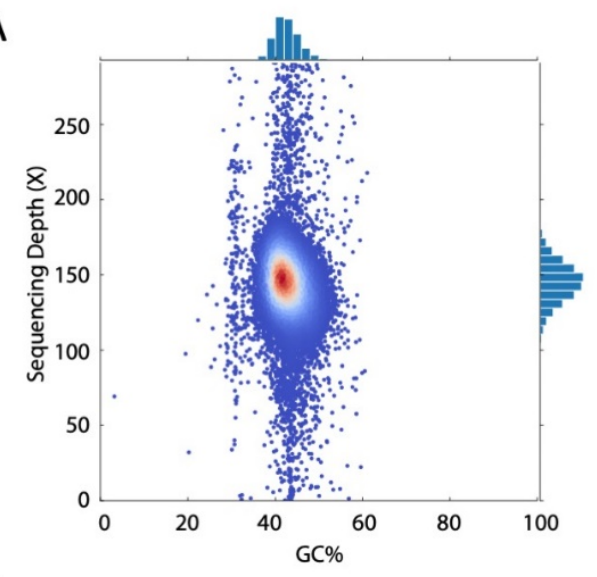

C

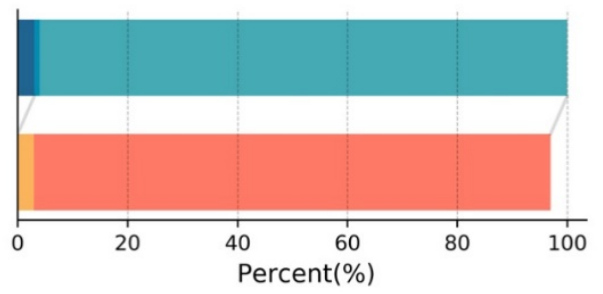

D
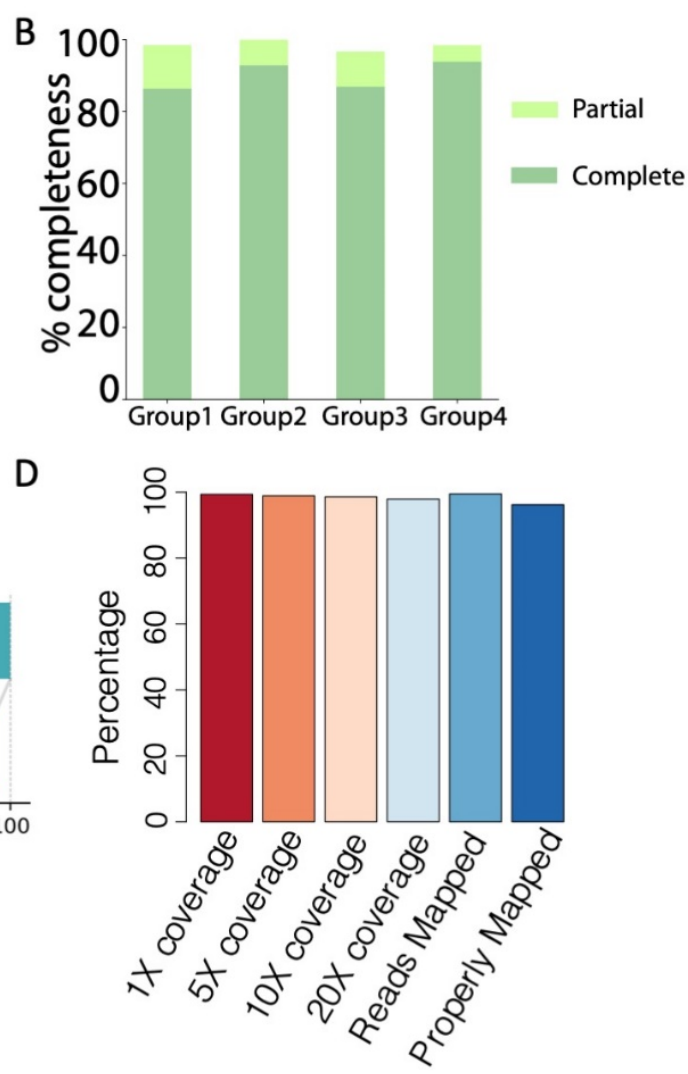

$E$

\begin{tabular}{|l|r|r|r|r|}
\hline & Contig Number & Contig Number ratio(\%) & Contig Length(bp) & Contig Length ratio(\%) \\
\hline On Metazoa & 45 & 72.58 & $536,062,546$ & 98.79 \\
\hline No hit & 17 & 27.42 & $6,592,183$ & 1.21 \\
\hline Total & 62 & 100 & $542,654,729$ & 100 \\
\hline
\end{tabular}

Figure 2. Quality evaluation of genome assembly. (A) GC content and sequencing depth distribution. (B) CEGMA evaluation. (C) BUSCO evaluation. (D) Illumina read mappability and genome coverage. The assembled genome is $99.35 \%$ covered at least once by Illumina reads, and $97.93 \%$ is covered at least 20 times. In addition, $99.48 \%$ of the Illumina reads were mapped to the assembled genome, and $96.22 \%$ were properly mapped (mapped paired reads with flag $0 \times 2$ set). (E) Genome contamination evaluation.

\subsection{Trachidermus fasciatus Genome Annotation}

We integrated de novo and RNA sequencing-based annotation methods for Trachidermus fasciatus genome. Based on PASA [31] gene structure annotation, the AUGUSTUS [33] de novo protein-coding gene prediction, and GeMoMa [32] homolog gene prediction, 14,238; 25,741; and 22,211 genes were identified, respectively. Evidence Modeler (EVM) [30] was used to integrate the genes from these three methods, and 23,191 protein-coding transcripts were finally identified by EVM. For transcript assembly, 35,392 transcripts of 17,867 genes were assembled by StringTie [35]. By integrating the results from EVM and StringTie, 19,147 protein genes with 35,093 transcripts (mRNAs) were found, which were used as final annotation for the protein-coding genes of Trachidermus fasciatus. In addition to protein-coding genes, 5572 rRNA, 2149 small RNA, and 7816 tRNA non-protein-coding genes were identified (Figure 4A). Compared with the genomes of the five closest species of Trachidermus fasciatus with an available annotated genome, no abnormal length distribution was observed for CDS, genes, exons, and introns (Figure 4B). Furthermore, the repetitive elements were also annotated. In total, $23.7 \%$ of the Trachidermus fasciatus genome was covered by repetitive elements, including LTR (4\%), LINE (6.4\%), SINE (0.6\%), and DNA repeats $(7 \%$ ) (Figure 4C). There are approximately 157,000 LINE elements, 526,000 DNA elements, 166,000 LTR elements, and 30,000 SINE elements (Figure 4D). 
A

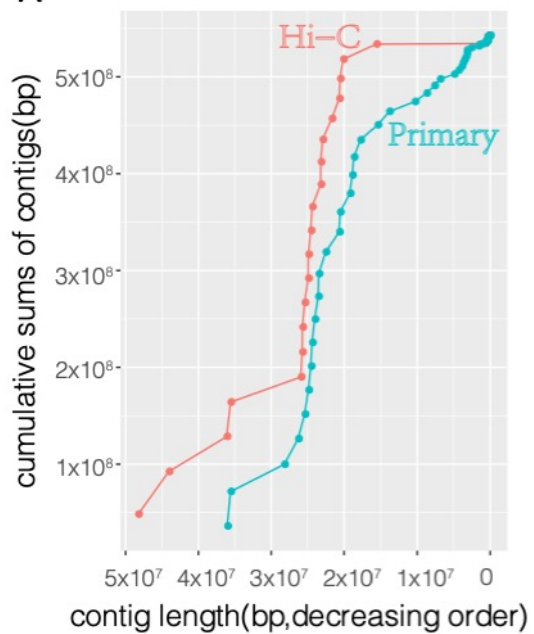

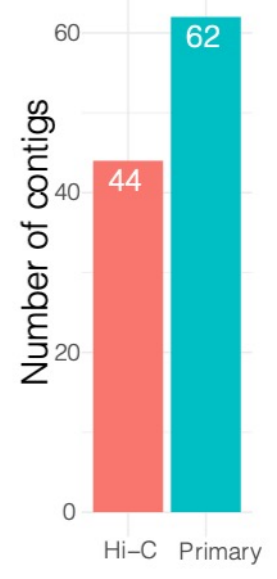

C

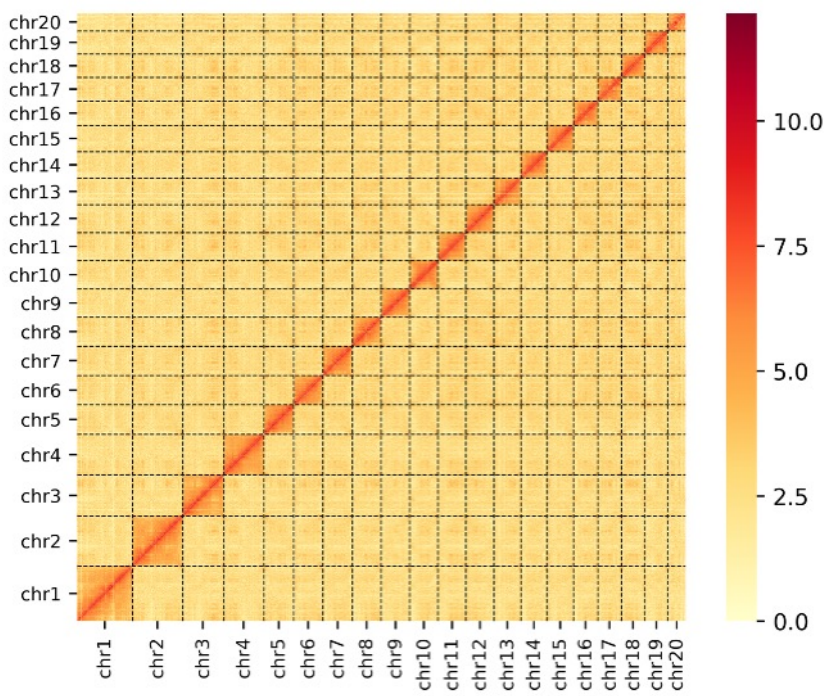

D

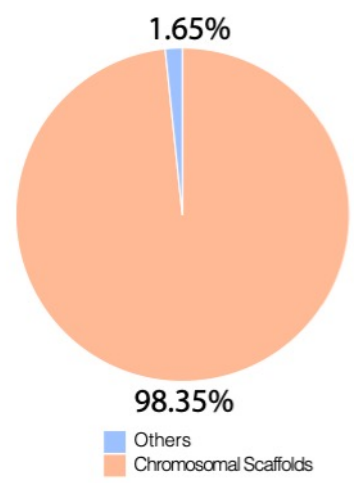

$E$

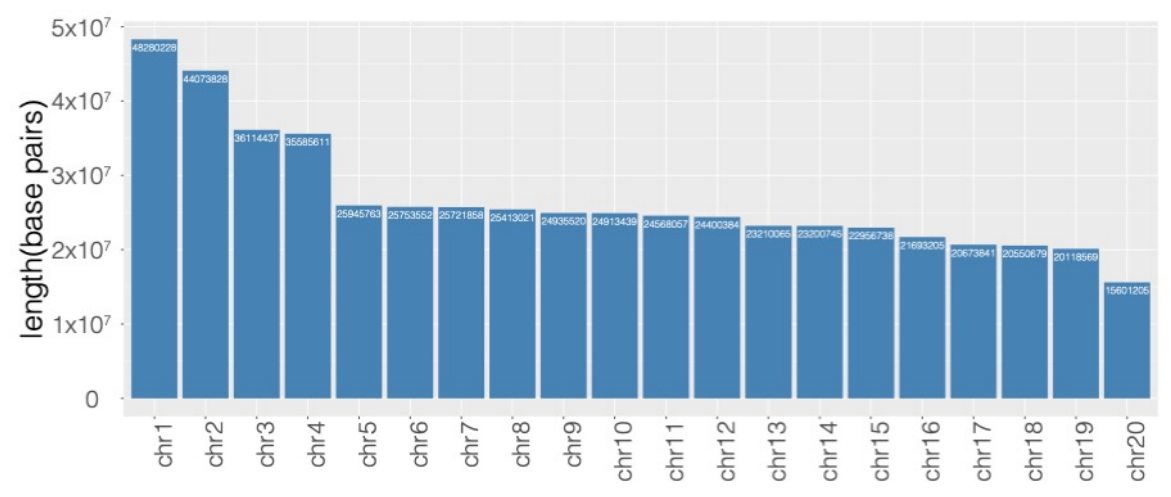

Figure 3. Hi-C enhanced genome assembly. (A) Comparison of cumulative sums of the contigs between the primary assembled genome and the Hi-C enhanced genome. (B) Reduced number of scaffolds/contigs in Hi-C enhanced genome. (C) Chromosomal level all-by-all Hi-C interaction heatmap (legend value is the natural log transformed linkage intensity).

(D) Genomic percentage of the chromosome-level scaffolds. (E) Length distribution for each chromosome.

\subsection{Novel Genes and Transcripts Identified}

As the first genome assembly for Trachidermus fasciatus, novel genes and transcripts were identified through RNR (RefSeq-NT-RNASeq integrated novel gene selection) strategy (Figure 5A). At the first step, transcript sequences were aligned onto the RefSeq sequences of four model organisms: human, mouse, zebrafish, and nematode. Among 35,093 proteincoding transcripts identified in this study, 6101 transcripts were failed to be aligned to RefSeq sequences of any selected organism. At the second step, a larger nucleotide sequence database (NCBI nucleotide database, or NT database), containing 63,454,572 sequences (genome and transcript sequences) from GenBank, EMBL, DDBJ and NCBI WGS, was used to find novel transcripts. Among 6101 transcript that failed to be mapped to any RefSeq sequence of selected organism, 5364 transcripts can be aligned to NT sequences, and 737 transcripts were unmappable to any of NT sequence. At the third step, detected RNA-seq reads ( $>50$ reads) were further required to select novel transcripts, which led to 82 transcripts defined as novel transcripts. Those 82 transcripts were associated with 64 genes, and among those genes, 51 were defined as novel genes based on two criteria: firstly, all the transcripts of those genes should be unmappable to either RefSeq sequences or NT sequences, and secondly, at least one of their transcripts had detected RNA-seq reads (>50 reads). 
A

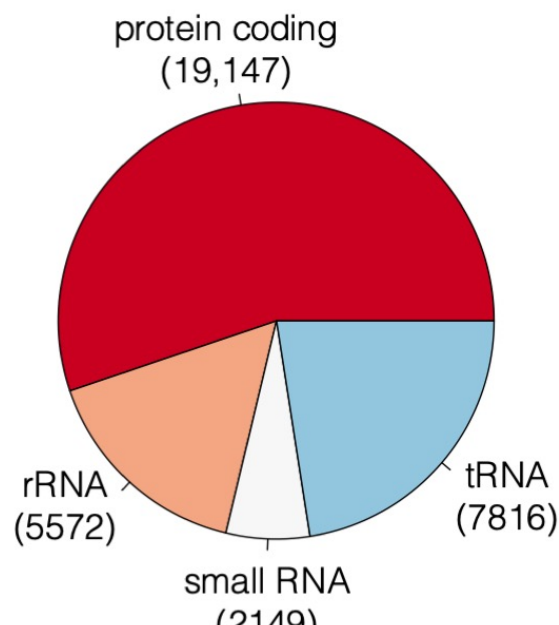

(2149)

C

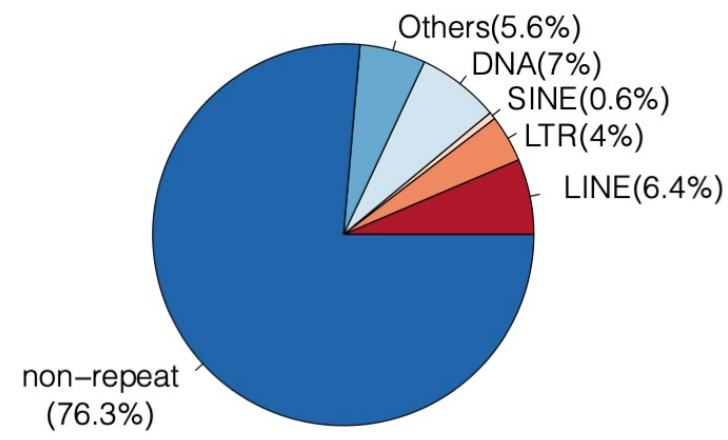

B

D
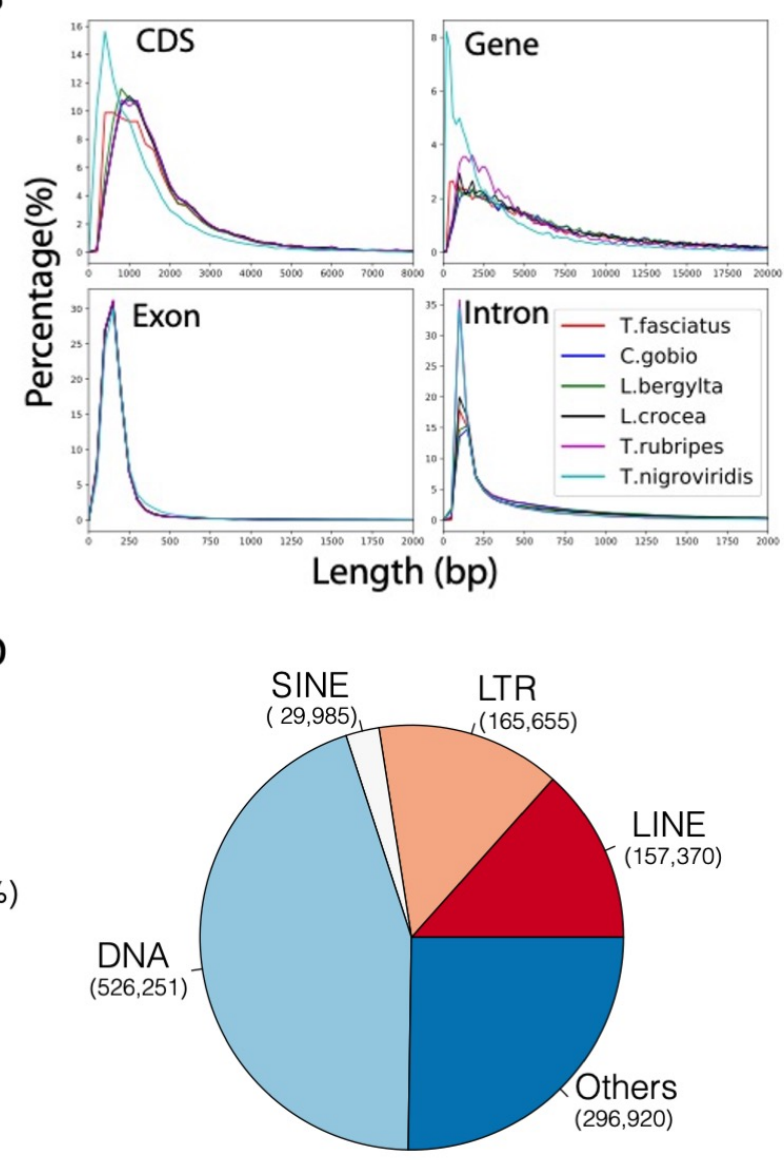

Figure 4. Summary of genome annotation for Trachidermus fasciatus. (A) Number of protein-coding and non-protein-coding genes. (B) Gene annotation length distribution compared between close species. (C) Percentage of genome occupied by repetitive elements. (D) Number of repetitive elements in each class.

Next, the RNA-seq expression levels were examined for 82 novel transcripts identified in this study (Figure 5B). Based on the expression profile of the seven tissues (liver, gall bladder, stomach, heart, kidney, gill, and skin), some novel transcript showed tissuespecific expression pattern. Among those tissue-specific expressed novel transcripts, the skin-specifically-expressed novel gene Trf.20428 was studied in detail. Trf.20428 contains three isoforms, and all of them were found to be as novel transcripts. As illustrated in the Sashimi plot based on the RNA-seq bam files (Figure S1), Trf.20428 showed specific expression in skin compared to other tissues studied, and the intron-exon boundaries of this transcript can be clearly identified by the mapped reads.

Furthermore, the protein sequences of the novel transcripts were mapped to NCBI NR database by blastp [42], and the protein sequences of 36 novel transcripts cannot be aligned to NR protein sequences under blastp expected threshold 0.05 (Figure S2A). For the 46 protein sequences that have mapped hits in the NR, the majority of them have an identity percentage with their best hits less than $60 \%$ (Figure S2B). The RNA-seq mapping detail for one of the blastp unmappable gene named Trf.5711 (with one transcript Trf.5711.1) was illustrated in Figure S3, which was highly expressed in all seven selected tissues. In summary, 82 transcripts and 51 genes (Tables S2 and S3, and sequence information in Supplementary File 1) from Trachidermus fasciatus were identified as novel transcripts and genes, respectively, and 36 novel transcripts contained protein sequences with no matched known sequences. 
A

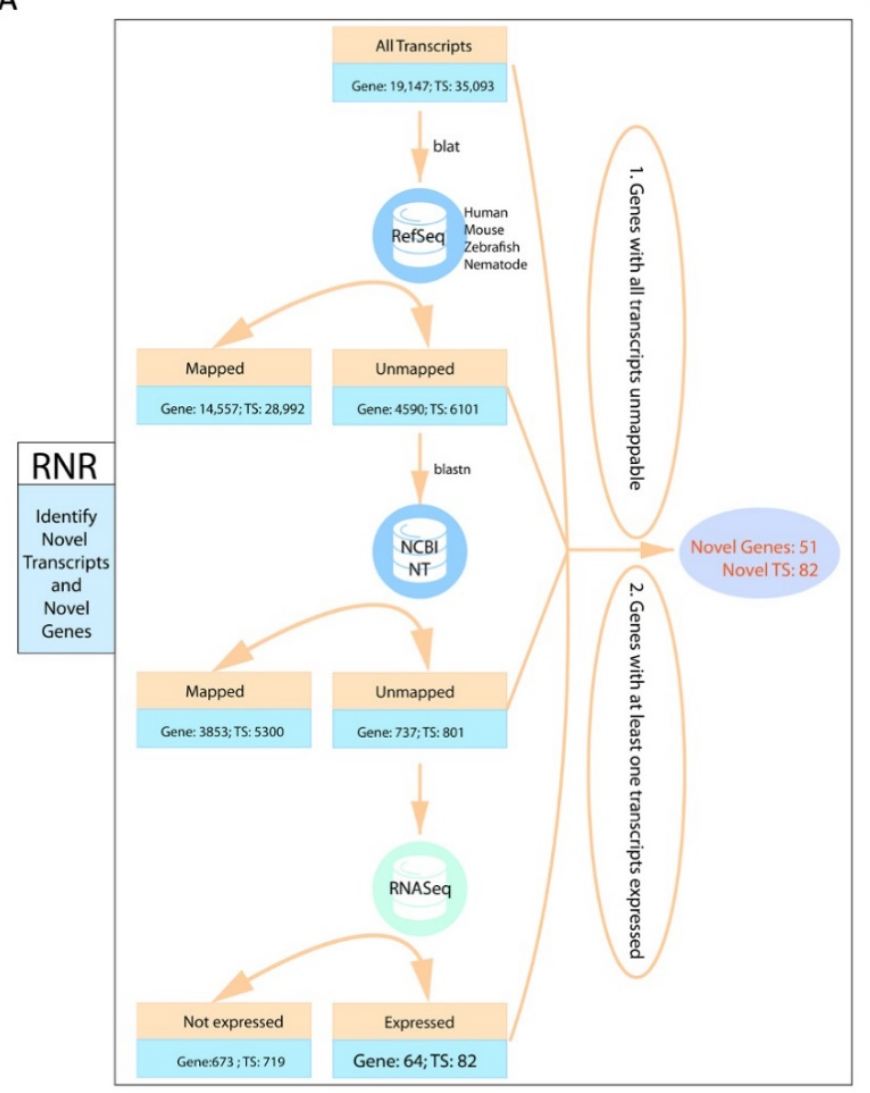

B

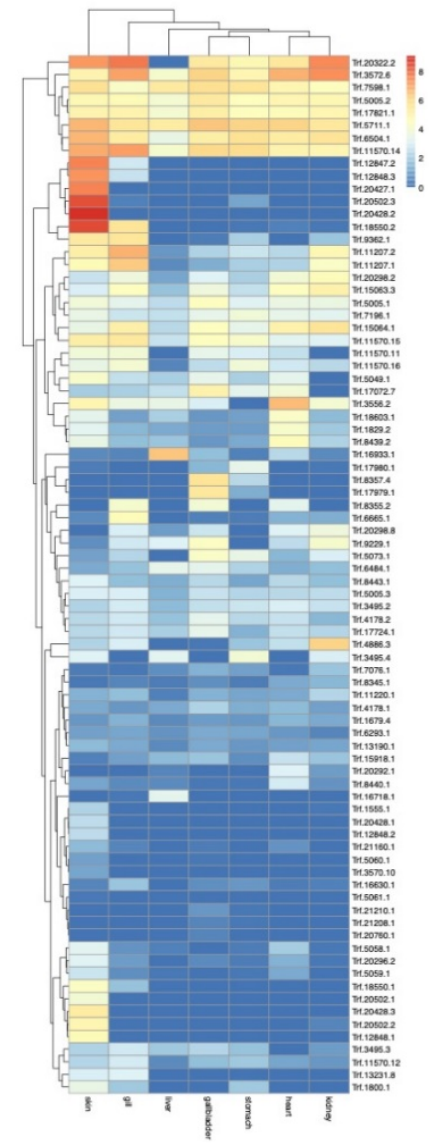

Figure 5. Identification of novel genes and transcripts. (A) Flowchart of RNR novel gene/transcript identification method. (B) Heatmap of the expression $(\log 1 \mathrm{p}(\mathrm{FPKM}))$ of the novel transcripts in seven tissues.

\section{Discussion}

In this study, we provided the first complete genome assembly for Trachidermus fasciatus (roughskin sculpin). Due to overfishing and environmental destruction, the population of roughskin sculpin is currently under threat in China, even though it has long been listed as a Class II protected animal. Our study might be important for future research on the protection and domesticated culturing of Trachidermus fasciatus. We not only provided the first genome reference for this species but also predicted its gene structures and annotated a total of 19,147 protein-coding genes. Genome annotation is important for future genetic studies on roughskin sculpin, which might provide a rich genetic resource for phenotypical and ecological studies. In addition to the genome assembly and genome annotation, in this study, we created a new method named RNR that can be used to identify novel transcripts/genes for a new species. Notably, we found 51 novel genes and 82 novel transcripts, which is useful to expand our knowledge about current gene pools from different species.

At the stage of preliminary assembly, we got 62 contigs with a combined length of $539,115,043 \mathrm{bp}$. Multiple factors could contribute to the small number of contigs at this stage: firstly, the estimated genome size of the species Trachidermus fasciatus is relatively smaller compared to the species such as zebrafish (1679M, GRCz11/danRer11), mouse (2730M, GRCm38/mm10), and human (3257M, GRCh38/hg38); secondly, the sequencing depth for both of Illumina sequencing and Nanopore sequencing is quite high $(100 \times$ and $160 \times$ respectively, Table 3 ); thirdly, the average length of nanopore reads is $21,384 \mathrm{bp}$, which could facilitate the assembly of contigs. Furthermore, based on the relationship (Table S4) between estimated chromosomes and the contigs of preliminary assembly (polished), the 
longest contigs from preliminary assembly occupies $99.798 \%$ of the estimated chromosome (chr3), which suggested that some of the preliminary contigs have achieved chromosomelevel assembly.

To evaluate the accuracy of the assembled genome at single-nucleotide level, we mapped the Illumina sequencing reads onto the assembled genome and calculated the accuracy based on the proportions of SNPs and INDELS from the mapping results. However, this naïve method still has a few limitations: we did not consider the sequencing errors generated by Illumina sequencing method and also ignored the mapping errors (especially for repetitive elements).

RNR method proposed in our study contains three levels of gene filtering, model organism RefSeq filtering, NCBI NT database filtering, and RNA-seq expression level filtering. Although it is possible to directly map our target sequences onto NCBI NT database, it will be computationally time consuming due to a large size of NT database (63 million sequences). In RNR, we suggest mapping the target sequences onto selected model organisms (with better genome assembly and gene annotation compared to nonmodel organisms), which would greatly reduce the number of target sequences for NT mapping.

In the previous study, the mitochondrial genome of Trachidermus fasciatus had been assembled [9]; thus, our study mainly focused on the nuclear genome assembly. To examine the mtDNA contamination in the nuclear genome assembly, we used blat (default setting) to map mtDNA sequence (NCBI Reference Sequence: NC_018770.1) onto our nuclear genome assembly, and no hit was found, which indicates low possibility of mtDNA contamination to our nuclear genome assembly.

At last, although we made the genome assembly and gene annotation publicly available, the genome browser tools to navigate the genome of Trachidermus fasciatus are still lacking. In the future, we will develop a genome browser to improve the accessibility of the genome-wide information for this species.

\section{Conclusions}

In this study, through combining nanopore sequencing and $\mathrm{Hi}-\mathrm{C}$ technologies, we assembled the first chromosomal-level high quality genome assembly for Trachidermus fasciatus. Furthermore, we proposed RNR method for novel genes finding, and in total 51 genes of Trachidermus fasciatus annotated in this study were identified as novel genes. Our study could greatly facilitate future studies for Trachidermus fasciatus.

Supplementary Materials: Available on https:/ / www.mdpi.com/article/10.3390/genes12050692/ s1. Figure S1: Sashimi plot illustration of skin specific expression of novel gene Trf.20428; Figure S2: (A) Mappability of novel transcripts protein sequences. Novel transcripts derived protein sequences were mapped to NCBI NR database by Blastp. (B) Distribution of blastp identity percentage for mappable novel transcripts derived protein sequences; Figure S3: Sashimi plot of gene Trf.5711 (Transcript ID Trf.5711.1), a novel transcript with unmappable protein sequence. The exon-exon junctional linkages and exon-level reads coverages were illustrated. Table S1: Statistics about Hi-C enhanced genome assembly. Table S2: Expression of novel transcripts. Table S3. List of novel genes and transcripts. Table S4. Relationship between Hi-C assembly scaffolds and contigs of polished preliminary assembly. Supplementary File 1: Sequence of 82 predicted novel transcripts.

Author Contributions: Conceptualization, G.X., J.W. and D.L.; Data curation, X.Z., F.L. and M.S.; Formal analysis, G.X., X.Z. and F.L.; Funding acquisition, G.X., F.L. and D.L.; Investigation, H.H.; Methodology, G.X., X.Z. and H.H. and J.W.; Resources, J.W. and D.L.; Software, G.X; Supervision, G.X., J.W. and D.L; Validation, X.Z.; Writing-original draft, G.X.; Writing-review and editing, J.W. and D.L. All authors have read and agreed to the published version of the manuscript.

Funding: This study was supported by grants from the National Natural Science Foundation of China (31900484 received by Gangcai Xie; 2018YFA0801004 received by Dong Liu), Natural Science Foundation of Jiangsu Province (BK20190924 received by Gangcai Xie; BK20180048, and BRA2019278 received by Dong Liu), startup fund for doctoral research of Nantong Science and Technology College (NTKY-Dr2017001 received by Feng Lv), Jiangsu Qing and Lan project (2018), Nantong Municipal Science and Technology Program (JCZ18012 received by Feng Lv), and the Open Program of Key 
Laboratory of Cultivation and High-Value Utilization of Marine Organisms in Fujian Province (2019 fjsccq08 received by Feng Lv).

Institutional Review Board Statement: The study was conducted according to the guidelines of the Declaration of Helsinki, and approved by the Ethics Committee of Nantong University (approve number SYXK(SU)2019-0188 and date of approval: 2019-04-23). All animal experimentation was carried out in accordance with the NIH Guidelines for the care and use of laboratory animals (http:/ / oacu.od.nih.gov/regs/index.htm) and ethically approved by the Administration Committee of Experimental Animals, Jiangsu Province, China [Approval ID: SYXK (SU) 2019-0188].

Informed Consent Statement: Not applicable.

Data Availability Statement: The genome sequence and genome annotation of Trachidermus fasciatus has been stored in the Genome Warehouse of National Genomics Data Center, Beijing Institute of Genomics (BIG), Chinese Academy of Sciences, under accession number GWHACFF00000000.2, which is publicly accessible at https://bigd.big.ac.cn/gwh.

Conflicts of Interest: The authors declare that there is no conflict of interest.

\section{References}

1. Wang, J.; Chen, G. The historical variance and causes of geographical distribution of a roughskin sculpin (Trachidermus fasciatus Heckel) in Chinese territory. Acta Ecol. Sin. 2010, 30, 6845-6853.

2. Islam, M.S.; Hibino, M.; Tanaka, M. Distribution and diet of the roughskin sculpin, Trachidermus fasciatus, larvae and juveniles in the Chikugo River estuary, Ariake Bay, Japan. Ichthyol. Res. 2007, 54, 160-167. [CrossRef]

3. Wang, J. Advances in studies on the ecology and reproductive biology of Trachidermus Fasciatus Heckel. Acta Hydrobiol. Sin. 1999, 23, 729-734.

4. Cao, L.; Wang, W.; Yang, C.; Wang, Y. Threatened fishes of the world: Trachidermus fasciatus Heckel, 1837 (Cottidae). Environ. Biol. Fishes 2008, 86, 63-64. [CrossRef]

5. Jinqiu Wang, W.L.; Qiang, J. Construction of healthy culture system of Roughskin sculpin. Fish. Mod. 2010, 37, 34-36.

6. Jianhua Chen, Z.Z.; Kunbao, L. Karyotype of Trachidermus Fasciatus Heckel. Zool. Res. 1984, 5, 103-104.

7. Li, Y.-L.; Xue, D.-X.; Gao, T.-X.; Liu, J.-X. Genetic diversity and population structure of the roughskin sculpin (Trachidermus fasciatus Heckel) inferred from microsatellite analyses: Implications for its conservation and management. Conserv. Genet. 2016, 17, 921-930. [CrossRef]

8. Li, Y.L.; Xue, D.X.; Zhang, B.D.; Liu, J.X. Population genomic signatures of genetic structure and environmental selection in the catadromous roughskin sculpin trachidermus fasciatus. Genome Biol. Evol. 2019, 11, 1751-1764. [CrossRef]

9. Zeng, Z.; Liu, Z.Z.; Pan, L.D.; Tang, S.J.; Wang, C.T.; Tang, W.Q.; Yang, J.Q. Complete mitochondrial genome of the endangered roughskin sculpin Trachidermus fasciatus (Scorpaeniformes, Cottidae). Mitochondrial DNA 2012, 23, 435-437. [CrossRef] [PubMed]

10. Reuter, J.A.; Spacek, D.V.; Snyder, M.P. High-throughput sequencing technologies. Mol. Cell 2015, 58, 586-597. [CrossRef] [PubMed]

11. Mardis, E.R. DNA sequencing technologies: 2006-2016. Nat. Protoc. 2017, 12, 213-218. [CrossRef]

12. Loman, N.J.; Quick, J.; Simpson, J.T. A complete bacterial genome assembled de novo using only nanopore sequencing data. Nat. Methods 2015, 12, 733-735. [CrossRef] [PubMed]

13. Jain, M.; Koren, S.; Miga, K.H.; Quick, J.; Rand, A.C.; Sasani, T.A.; Tyson, J.R.; Beggs, A.D.; Dilthey, A.T.; Fiddes, I.T.; et al. Nanopore sequencing and assembly of a human genome with ultra-long reads. Nat. Biotechnol. 2018, 36, 338-345. [CrossRef] [PubMed]

14. Bowden, R.; Davies, R.W.; Heger, A.; Pagnamenta, A.T.; de Cesare, M.; Oikkonen, L.E.; Parkes, D.; Freeman, C.; Dhalla, F.; Patel, S.Y.; et al. Sequencing of human genomes with nanopore technology. Nat. Commun. 2019, 10, 1869. [CrossRef]

15. Choi, J.Y.; Lye, Z.N.; Groen, S.C.; Dai, X.; Rughani, P.; Zaaijer, S.; Harrington, E.D.; Juul, S.; Purugganan, M.D. Nanopore sequencing-based genome assembly and evolutionary genomics of circum-basmati rice. Genome Biol. 2020, 21, 21. [CrossRef]

16. Burton, J.N.; Adey, A.; Patwardhan, R.P.; Qiu, R.; Kitzman, J.O.; Shendure, J. Chromosome-scale scaffolding of de novo genome assemblies based on chromatin interactions. Nat. Biotechnol. 2013, 31, 1119-1125. [CrossRef]

17. Belton, J.M.; McCord, R.P.; Gibcus, J.H.; Naumova, N.; Zhan, Y.; Dekker, J. Hi-C: A comprehensive technique to capture the conformation of genomes. Methods 2012, 58, 268-276. [CrossRef] [PubMed]

18. Hu, J.; Fan, J.; Sun, Z.; Liu, S. NextPolish: A fast and efficient genome polishing tool for long-read assembly. Bioinformatics 2020, 36, 2253-2255. [CrossRef] [PubMed]

19. Simao, F.A.; Waterhouse, R.M.; Ioannidis, P.; Kriventseva, E.V.; Zdobnov, E.M. BUSCO: Assessing genome assembly and annotation completeness with single-copy orthologs. Bioinformatics 2015, 31, 3210-3212. [CrossRef]

20. Parra, G.; Bradnam, K.; Korf, I. CEGMA: A pipeline to accurately annotate core genes in eukaryotic genomes. Bioinformatics 2007, 23, 1061-1067. [CrossRef] 
21. Kriventseva, E.V.; Kuznetsov, D.; Tegenfeldt, F.; Manni, M.; Dias, R.; Simao, F.A.; Zdobnov, E.M. OrthoDB v10: Sampling the diversity of animal, plant, fungal, protist, bacterial and viral genomes for evolutionary and functional annotations of orthologs. Nucleic Acids Res. 2019, 47, D807-D811. [CrossRef] [PubMed]

22. Li, H.; Durbin, R. Fast and accurate short read alignment with Burrows-Wheeler transform. Bioinformatics 2009, 25, 1754-1760. [CrossRef]

23. Danecek, P.; McCarthy, S.A. BCFtools/csq: Haplotype-aware variant consequences. Bioinformatics 2017, 33, 2037-2039. [CrossRef] [PubMed]

24. Li, H. Minimap2: Pairwise alignment for nucleotide sequences. Bioinformatics 2018, 34, 3094-3100. [CrossRef] [PubMed]

25. Altschul, S.F.; Gish, W.; Miller, W.; Myers, E.W.; Lipman, D.J. Basic local alignment search tool. J. Mol. Biol. 1990, 215 , 403-410. [CrossRef]

26. Chen, S.; Zhou, Y.; Chen, Y.; Gu, J. Fastp: An ultra-fast all-in-one FASTQ preprocessor. Bioinformatics 2018, 34, i884-i890. [CrossRef] [PubMed]

27. Tarailo-Graovac, M.; Chen, N. Using RepeatMasker to identify repetitive elements in genomic sequences. Curr. Protoc. Bioinform. 2009. [CrossRef]

28. Han, Y.; Wessler, S.R. MITE-Hunter: A program for discovering miniature inverted-repeat transposable elements from genomic sequences. Nucleic Acids Res. 2010, 38, e199. [CrossRef]

29. Bao, W.; Kojima, K.K.; Kohany, O. Repbase Update, a database of repetitive elements in eukaryotic genomes. Mob. DNA 2015, 6, 11. [CrossRef]

30. Haas, B.J.; Salzberg, S.L.; Zhu, W.; Pertea, M.; Allen, J.E.; Orvis, J.; White, O.; Buell, C.R.; Wortman, J.R. Automated eukaryotic gene structure annotation using EVidenceModeler and the program to assemble spliced alignments. Genome Biol. 2008,9, R7. [CrossRef]

31. Haas, B.J.; Delcher, A.L.; Mount, S.M.; Wortman, J.R.; Smith, R.K., Jr.; Hannick, L.I.; Maiti, R.; Ronning, C.M.; Rusch, D.B.; Town, C.D.; et al. Improving the Arabidopsis genome annotation using maximal transcript alignment assemblies. Nucleic Acids Res. 2003, 31, 5654-5666. [CrossRef] [PubMed]

32. Keilwagen, J.; Hartung, F.; Paulini, M.; Twardziok, S.O.; Grau, J. Combining RNA-seq data and homology-based gene prediction for plants, animals and fungi. BMC Bioinform. 2018, 19, 189. [CrossRef]

33. Stanke, M.; Diekhans, M.; Baertsch, R.; Haussler, D. Using native and syntenically mapped cDNA alignments to improve de novo gene finding. Bioinformatics 2008, 24, 637-644. [CrossRef] [PubMed]

34. Kim, D.; Paggi, J.M.; Park, C.; Bennett, C.; Salzberg, S.L. Graph-based genome alignment and genotyping with HISAT2 and HISAT-genotype. Nat. Biotechnol. 2019, 37, 907-915. [CrossRef]

35. Pertea, M.; Pertea, G.M.; Antonescu, C.M.; Chang, T.C.; Mendell, J.T.; Salzberg, S.L. StringTie enables improved reconstruction of a transcriptome from RNA-seq reads. Nat. Biotechnol. 2015, 33, 290-295. [CrossRef]

36. Pertea, G.; Pertea, M. GFF Utilities: GffRead and GffCompare. F1000Res 2020, 9. [CrossRef]

37. Nawrocki, E.P.; Eddy, S.R. Infernal 1.1: 100-fold faster RNA homology searches. Bioinformatics 2013, 29, 2933-2935. [CrossRef]

38. Lowe, T.M.; Eddy, S.R. tRNAscan-SE: A program for improved detection of transfer RNA genes in genomic sequence. Nucleic Acids Res. 1997, 25, 955-964. [CrossRef] [PubMed]

39. Wang, M.; Kong, L. Pblat: A multithread blat algorithm speeding up aligning sequences to genomes. BMC Bioinform. 2019, 20, 28. [CrossRef]

40. Boratyn, G.M.; Camacho, C.; Cooper, P.S.; Coulouris, G.; Fong, A.; Ma, N.; Madden, T.L.; Matten, W.T.; McGinnis, S.D.; Merezhuk, Y.; et al. BLAST: A more efficient report with usability improvements. Nucleic Acids Res. 2013, 41, W29-W33. [CrossRef]

41. Patro, R.; Duggal, G.; Love, M.I.; Irizarry, R.A.; Kingsford, C. Salmon provides fast and bias-aware quantification of transcript expression. Nat. Methods 2017, 14, 417-419. [CrossRef] [PubMed]

42. Camacho, C.; Coulouris, G.; Avagyan, V.; Ma, N.; Papadopoulos, J.; Bealer, K.; Madden, T.L. BLAST+: Architecture and applications. BMC Bioinform. 2009, 10, 421. [CrossRef] [PubMed]

43. Garrido-Martin, D.; Palumbo, E.; Guigo, R.; Breschi, A. Ggsashimi: Sashimi plot revised for browser- and annotation-independent splicing visualization. PLoS Comput. Biol. 2018, 14, e1006360. [CrossRef]

44. National Genomics Data Center Members and Partners. Database resources of the national genomics data center in 2020. Nucleic Acids Res. 2020, 48, D24-D33. 